\title{
Desempenho auditivo ao longo do tempo em usuários de implante coclear com perda auditiva pós-lingual
}

\author{
Auditory performance, during a period of time in cochlear \\ implant users with post lingual hearing loss
}

\begin{abstract}
Laísa Flávia Soares Fernandes Peixoto Buarque', Joseli Soares Brazorotto², Hannalice Gottschalck Cavalcanti, Luiz Rodolpho Penna Lima Júnior', Danielle do Vale Silva Penna Lima ${ }^{4}$, Maria Ângela Fernandes Ferreira ${ }^{5}$
\end{abstract}

\begin{abstract}
RESUMO
Objetivo: Avaliar o desempenho auditivo ao longo do tempo e investigar os fatores prognósticos relacionados. Métodos: Estudo de coorte retrospectivo realizado por meio da análise dos prontuários de 57 indivíduos com perda auditiva pós-lingual e usuários de implante coclear há pelo menos 12 meses. Utilizou-se o estimador de Kaplan-Meier e o teste de Logrank para análise da percepção da fala ao longo do tempo e da possível interferência de fatores como gênero, escolaridade, tempo de privação auditiva, idade ao implante, marca do dispositivo e etiologia, sobre o desfecho estudado. Resultados: Em apenas 12 meses após a ativação do implante, mais da metade da coorte já apresentava escores satisfatórios no teste de percepção da fala. O teste de Logrank não indicou diferença entre as covariáveis testadas. Houve diferença significativa entre os resultados auditivos pré e pós- implante coclear. Conclusão: $\mathrm{O}$ desempenho auditivo, após 12 meses de uso do IC, superou o critério de indicação na maioria dos indivíduos com perda auditiva pós-lingual, independentemente dos fatores prognósticos avaliados, porém não em todos os indivíduos pesquisados.
\end{abstract}

Descritores: Implante coclear; Perda auditiva neurossensorial; Percepção da fala; Prognóstico; Análise de sobrevivência

\begin{abstract}
Purpose: Evaluating the hearing accomplishment over time and to investigate the prognostic factors related. Methods: A retrospective cohort study carried out through analysis the medical records of 57 patients with post-lingual hearing loss and cochlear implant for at least 12 months. To sustain our method we applied the Kaplan-Meier method and the logrank test for analysis of speech perception over time and the possible influence of factors such as gender, education, duration of hearing loss, age at implantation, etiology and device's brand, on the outcome studied. Results: In only 12 months after implant activation, more than half of the cohort already had satisfactory scores on the test of speech perception. The logrank test had not indicated difference among the covariates tested. There was a significant difference between the hearing results pre and post cochlear implant. Conclusion: The hearing performance after 12 months using the IC exceeds the criterion for the majority of individuals with post-lingual hearing loss, regardless of prognostic factors evaluated, but not all respondents.
\end{abstract}

Keywords: Cochlear implant; Hearing loss, Sensorineural; Speech perception; Prognosis; Survival analysis

Trabalho realizado no Programa de Pós-Graduação em Saúde Coletiva, Universidade Federal do Rio Grande do Norte - UFRN - Natal (RN), Brasil, com bolsa concedida pela Coordenação de Aperfeiçoamento de Pessoal de Nível Superior (CAPES).

(1) Programa de Pós-graduação em Saúde Coletiva (Mestrado), Universidade Federal do Rio Grande do Norte - UFRN - Natal (RN), Brasil.

(2) Departamento de Fonoaudiologia, Universidade Federal do Rio Grande do Norte - UFRN - Natal (RN), Brasil.

(3) Departamento de Fonoaudiologia, Universidade Federal da Paraíba - UFPB - João Pessoa (PB), Brasil.

(4) Programa de Implante Coclear do Otocentro/ Hospital do Coração de Natal, Natal (RN), Brasil.

(5) Departamento de Odontologia, Universidade Federal do Rio Grande do Norte - UFRN - Natal (RN), Brasil.

Conflito de interesses: Não

Contribuição dos autores: $L F S F P B$ pesquisador principal, elaboração da pesquisa, elaboração do cronograma, levantamento da literatura, coleta, análise dos dados, redação do artigo, submissão e trâmites do artigo; JSB e MAFF orientadores, elaboração da pesquisa, elaboração do cronograma, análise dos dados, correção da redação do artigo, aprovação da versão final; $H G C, L R P L J$ e DVSPL pesquisadores auxiliares, elaboração da pesquisa, coleta de dados e redação do artigo. Endereço para correspondência: Laísa Flávia Soares Fernandes Peixoto Buarque. Av. Senador Salgado Filho, 1787, Lagoa Nova, Natal (RN), Brasil, CEP: 59056-000. E-mail: laisabuarque@gmail.com

Recebido em: 18/3/2013; Aceito em: 3/7/2013 


\section{INTRODUÇÃO}

Em pacientes com perda auditiva pós-lingual, a utilização do Implante Coclear (IC) busca a restauração da percepção auditiva e a comunicação oral ${ }^{(1)}$. Os resultados auditivos obtidos por indivíduos com perda auditiva pós-lingual têm sido de grande interesse, uma vez que a experiência auditiva prévia permite avaliar o desempenho auditivo antes e depois do implante coclear e, assim, determinar o benefício alcançado com o uso desse dispositivo ${ }^{(2)}$.

Uma das estratégias para avaliar esse benefício é a aplicação de testes de percepção da fala. Esses testes empregam listas de vogais, sílabas, palavras e sentenças em apresentação aberta e sem uso de pistas contextuais e fechadas. Os escores gerados permitem avaliar a evolução no uso do IC e devem ser realizados ao longo do acompanhamento pós-operatório.

Dentre os fatores relatados na literatura que podem interferir nos resultados auditivos, destacam-se, o período de aquisição da surdez, o tempo de privação sensorial, a integridade das vias auditivas centrais, a habilidade de leitura orofacial, o número de eletrodos inseridos na cóclea, a etiologia da surdez, a qualidade e a tecnologia do dispositivo e o tipo e a qualidade $\mathrm{d}$ treinamento oferecido a um implantado ${ }^{(3-5)}$.

Predizer o desempenho na percepção da fala em candidatos ao IC tem sido, por longo tempo, o objetivo de clínicos e pesquisadores ${ }^{(6)}$. O tempo em que o benefício é alcançado, é, sem dúvida, um fator importante no aconselhamento dos pacientes candidatos à cirurgia. Entretanto, na literatura, há divergências sobre esse período ${ }^{(7,8)}$.

O presente estudo visou avaliar o desempenho auditivo ao longo do tempo em usuários de IC adultos com perdas auditivas pós-linguais, investigando possíveis fatores prognósticos.

\section{MÉTODOS}

A população estudada consistiu em 57 usuários adultos (33 homens e 24 mulheres) que foram acompanhados, retrospectivamente, por meio da coleta no banco de dados do Programa de Implante Coclear de um centro de saúde auditiva, situado em Natal, Rio Grande do Norte. O acompanhamento da população compreendeu o período de 2000 a 2011. Os critérios de inclusão foram: indivíduos com perda auditiva neurossensorial de grau severo a profundo, adquirida no período pós-lingual, usuários de IC por um período mínimo de 12 meses.

Indivíduos com espectro da neuropatia auditiva, com outras deficiências sensoriais, com malformação de cóclea ou hipoplasia de nervo auditivo e cujos prontuários apresentavam informações incompletas sobre os dados audiológicos pré e/ou pós-implante coclear, foram excluídos da análise.

Para a caracterização da população e posterior análise estatística, as seguintes informações foram coletadas nos prontuários: gênero, escolaridade, tempo de privação auditiva, característica da surdez, etiologia, modelo do dispositivo, orelha implantada, idade ao implante e índice de acerto em testes de percepção da fala com sentenças em conjunto aberto no período pré e pós-implante coclear com suas respectivas datas de realização.

A análise retrospectiva da coorte foi realizada por meio dos dados coletados nos prontuários e observados os seus resultados auditivos aos 3, 6 e 12 meses após o IC. O Teste de Percepção da Fala (TPF) com sentenças utilizado nesta pesquisa foi desenvolvido pelo Centro de Pesquisas Audiológicas (CPA) - Hospital de Reabilitação de Anomalias Craniofaciais (HRAC) da Universidade de São Paulo (USP), Bauru ${ }^{(9)}$. Esse teste foi aplicado nas avaliações periódicas pré e pós-implante coclear, em ambiente silencioso, à viva voz e com o paciente posicionado a uma distância de um metro do avaliador.

No presente estudo, considerou-se satisfatório um índice superior a 50\% de acertos, pois admite-se que, para esses indivíduos, o implante coclear foi benéfico.

$\mathrm{O}$ teste de sentenças em conjunto aberto foi submetido à análise de sobrevida (teste estatístico que avalia o prognóstico de indivíduos após uma intervenção clínica) por meio do estimador de Kaplan-Meier para que o benefício do implante coclear fosse avaliado ao longo do tempo. A condição dicotômica para avaliação do desfecho foi "benefício esperado" para índices de acerto iguais ou superiores a 50\% (evento de interesse) e "sem benefício" para índices de acerto inferiores a $50 \%$ (censura).

Após a análise pelo estimador de Kaplan-Meier, o desfecho foi submetido à análise univariada por meio do teste de Logrank com os fatores preditores do desempenho auditivo, a saber, gênero, escolaridade, tempo de privação auditiva, idade ao implante, marca do dispositivo e etiologia.

A comparação entre os resultados auditivos antes e após 12 meses de uso do IC foi realizada por meio do teste $t$ para amostras emparelhadas.

A pesquisa foi aprovada pelo Comitê de Ética em Pesquisa da Universidade Federal do Rio Grande do Norte e obteve parecer favorável à sua realização, mediante processo de número 040/2012. Os pacientes foram informados sobre os procedimentos a serem realizados e assinaram um Termo de Consentimento Livre e Esclarecido, consentindo com a participação neste estudo, em atendimento à Resolução 196/96 do Conselho Nacional de Saúde.

\section{RESULTADOS}

Com relação à etiologia da perda auditiva, a causa desconhecida foi a mais prevalente, encontrada em $42,10 \%$ da população, seguida pelas causas genéticas $(14,0 \%)$, meningite $(12,30 \%)$ e o Traumatismo Crânio Encefálico (TCE), com $8,8 \%$ dos achados. Outras etiologias $(21,10 \%)$ incluíram a perda auditiva induzida por ruído, ototoxicidade, síndrome de Usher, trauma acústico, catapora, otite média crônica, doença de Menière e caxumba. A paciente com Síndrome de Usher 
apresentou também, como sintoma secundário, a retinose pigmentar. Entretanto, foi incluída na pesquisa, uma vez que sua acuidade visual estava conservada, encontrando-se, apenas no momento da análise, com o campo visual parcialmente comprometido.

As demais características da amostra referentes aos dados demográficos, perda auditiva e o modelo do implante coclear são apresentadas na Tabela 1 .

Tabela 1. Características dos indivíduos incluídos na pesquisa

\begin{tabular}{|c|c|c|c|}
\hline Características & $\mathrm{n}(\%)$ & Média & $\begin{array}{l}\text { Desvio- } \\
\text { padrão }\end{array}$ \\
\hline Idade ao implante* & & 45,31 & 17,73 \\
\hline Duração da perda auditiva* & & 17,31 & 14,13 \\
\hline \multicolumn{4}{|l|}{ Sexo } \\
\hline Masculino & $33(57,9)$ & & \\
\hline Feminino & $24(42,1)$ & & \\
\hline \multicolumn{4}{|l|}{ Escolaridade } \\
\hline Até 8 anos de estudo & $20(35,1)$ & & \\
\hline Acima de 8 anos de estudo & $37(64,9)$ & & \\
\hline \multicolumn{4}{|l|}{ Instauração da perda auditiva } \\
\hline Progressiva & $42(73,7)$ & & \\
\hline Súbita & $15(26,3)$ & & \\
\hline \multicolumn{4}{|l|}{ Modelo do Implante } \\
\hline \multicolumn{4}{|l|}{ Cochlear } \\
\hline \multicolumn{4}{|c|}{ Nucleus $₫ 24$ Contour Advanced $15(26,3)$} \\
\hline Nucleus $₫ 24$ Contour & $6(10,5)$ & & \\
\hline Nucleus $\circledast 24 \mathrm{~K}$ & $1(1,8)$ & & \\
\hline Nucleus $尺 24$ & $9(15,8)$ & & \\
\hline \multicolumn{4}{|l|}{ Med-El } \\
\hline Pulsar® Cl100 & $15(26,3)$ & & \\
\hline COMB 40 & $6(10,5)$ & & \\
\hline SONATAti 100 & $4(7,0)$ & & \\
\hline \multicolumn{4}{|l|}{ Advanced Bionics } \\
\hline HiRes $® 90 \mathrm{~K}$ & $1(1,8)$ & & \\
\hline \multicolumn{4}{|l|}{ Lado implantado } \\
\hline Direito & $18(31,6)$ & & \\
\hline Esquerdo & $39(68,4)$ & & \\
\hline
\end{tabular}

* Em anos

Foi evidenciado que o benefício alcançado pelo implante coclear ocorre nos primeiros meses de uso, ou seja, os indivíduos foram capazes de perceber os sons da fala, com índices de acerto de sentenças igual ou superior a $50 \%$ das apresentações. Observou-se que, aos 12 meses de uso, aproximadamente $60 \%$ da população já havia alcançado escores satisfatórios para os TPF com sentenças em conjunto aberto (Figura 1).

No resultado satisfatório para percepção da fala, 15,8\% dos indivíduos da amostra (9) não obtiveram o benefício esperado (evento de interesse). O teste de Logrank indicou que não houve

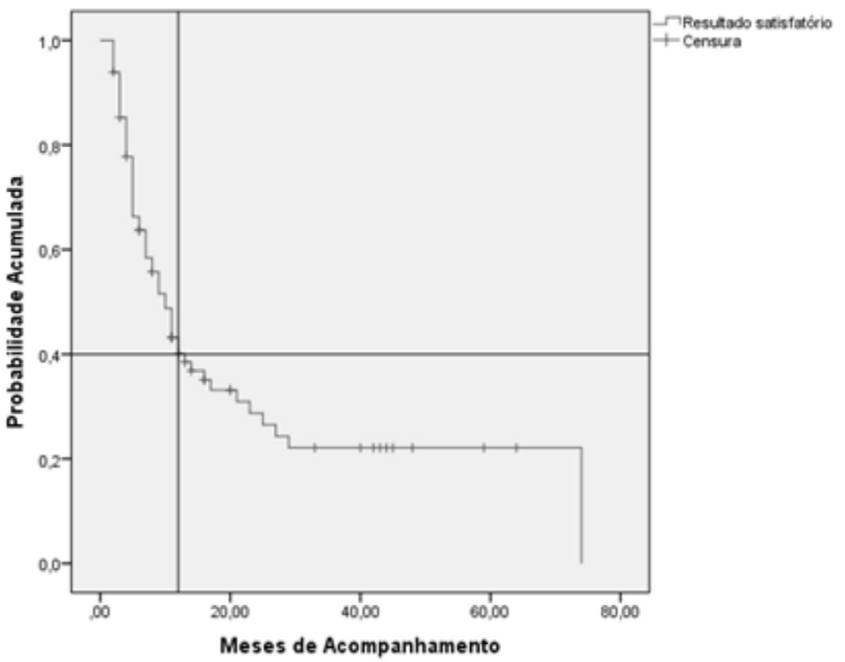

Figura 1. Curva da probabilidade acumulada por meio do estimador de Kaplan-Meier para percepção da fala satisfatória em usuários de IC acometidos com perda auditiva pós-lingual

diferença entre o tempo para percepção da fala e as variáveis independentes (Tabela 2).

Os resultados pré-implante coclear foram comparados aos resultados da audiometria tonal, representada pela média tritonal das frequências de 500, 1000 e $2000 \mathrm{~Hz}$ e dos testes de fala em conjunto aberto, aproximadamente 12 meses após a cirurgia do IC. Houve diferença $(\mathrm{p}<0,001)$ nas duas análises (Tabela 3).

\section{DISCUSSÃO}

O IC é o método atual mais adequado para adultos com surdez pós-lingual em grau severo-profundo. Os resultados do presente estudo mostram que o implante coclear é uma intervenção com efeitos benéficos para a detecção auditiva e para a percepção da fala dessa população. $\mathrm{O}$ benefício obtido manteve-se ao longo do período estudado. Outros estudos apontam para resultados que concordam com os nossos ${ }^{(1,10)}$.

Os mesmos resultados foram observados em uma população de idosos. Tais benefícios mantiveram-se ao longo do tempo em uma média de 44 meses de acompanhamento ${ }^{(10)}$.

Da mesma forma, indivíduos adultos com perda auditiva pós-lingual, acompanhados aos 3, 6 e 12 meses pós-implante coclear, também apresentaram melhoras significativas. Tais efeitos foram observados desde a primeira avaliação, aos 3 meses, aumentando, progressivamente, até os 12 meses $^{(1)}$.

Não foi observada diferença no desempenho entre homens e mulheres usuários de IC. Outros estudos também não relatam esse tipo de diferença entre os gêneros ${ }^{(2,11)}$.

A etiologia e a forma de instauração da perda auditiva (aparecimento súbito ou progressivo) não foram associadas aos resultados auditivos da percepção da fala, mesma constatação de outros autores ${ }^{(2,11,12)}$. O fato de a população estudada apresentar, em sua maioria, causa desconhecida, prejudicou a 
Tabela 2. Probabilidade acumulada para os índices satisfatórios dos Testes de Percepção da Fala de acordo com os fatores de prognóstico aos 3,6 e 12 meses de uso do implante coclear

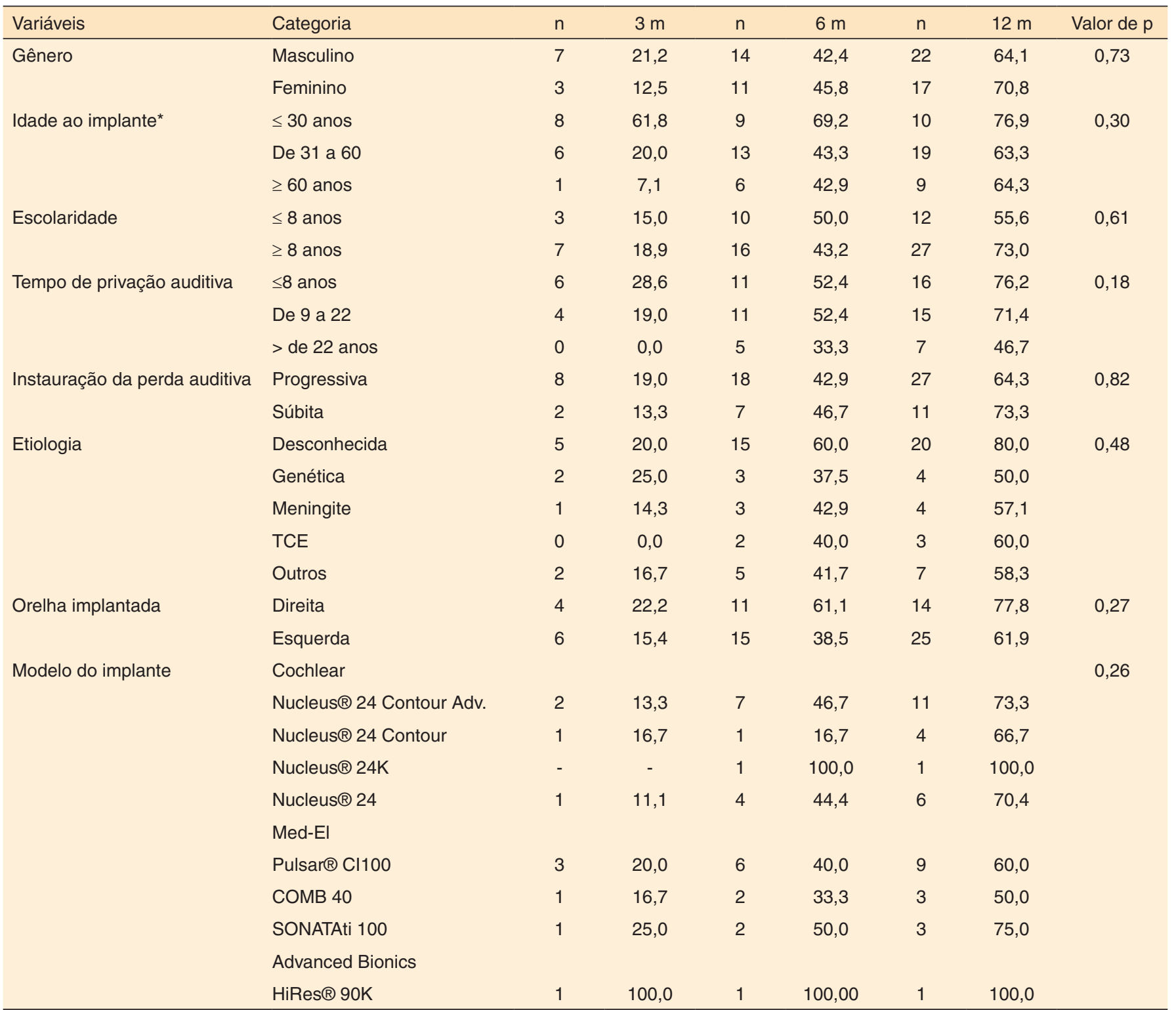

Teste de Logrank $(p \leq 0,05)$

* Categorizado em faixa etária

Tabela 3. Resultados da audiometria em campo livre e dos escores dos TPF com sentenças em conjunto aberto antes e após 12 meses de implante coclear

\begin{tabular}{lcccc}
\hline Desempenho auditivo & $1^{\text {a }}$ medida (DP) & $2^{\text {a }}$ medida (DP) & Teste t & Valor de $p$ \\
\hline Audiometria em campo livre (dB) & $105,26(15,79)$ & $33,42(9,31)$ & 28,96 & $<0,001^{*}$ \\
Teste de percepção da fala (\%) & $7,02(0,12)$ & $68,26(0,31)$ & $-15,10$ & $<0,001^{*}$
\end{tabular}

*Valores significativos $(p \leq 0,05)$ - Teste t para amostras emparelhadas

Legenda: DP = desvio-padrão

análise dos dados, na medida em que não havia informação sobre o verdadeiro motivo da perda auditiva. A etiologia pode prover dados sobre o grau de ossificação e má formação coclear e sobre o desenvolvimento do nervo auditivo, sendo, portanto, determinante nos resultados pós-cirúrgicos ${ }^{(13)}$.

No que se refere à forma do surgimento da perda auditiva, acreditamos que pacientes que sofrem perdas auditivas severas e súbitas procuram auxílio médico mais rapidamente do que aqueles que sofrem de perdas auditivas progressivas, possibilitando, com isso, o início imediato da reabilitação auditiva. Já aqueles que têm perdas auditivas progressivas, fazem uso de próteses auditivas até indicação da cirurgia. Dessa forma, 
acreditamos que a deterioração das vias auditivas centrais também pode ser amenizada.

A faixa etária e o tempo de privação auditiva não foram associados ao desempenho. Em alguns estudos, foi observado que o fator mais influente para o resultado auditivo não é a idade por ocasião do implante e sim o tempo que o paciente permaneceu com perda auditiva ${ }^{(2,7,11,12,14,15)}$. O sucesso do IC requer a funcionalidade do processamento da via auditiva intacta, desde as células do gânglio espiral até o córtex auditivo ${ }^{(16)}$. A deterioração dessa via está mais relacionada ao tempo de privação auditiva do que a idade em que o sujeito foi implantado. Esse fato explicaria a importância de um período curto de privação auditiva para um bom desempenho do $\mathrm{IC}^{(14)}$.

Apesar de a explicação parecer bastante convincente, muitos outros estudos, diversamente do nosso, não confirmaram essa premissa, não encontrando qualquer associação com o tempo de privação auditiva ${ }^{(1,4,15,17)}$.

Os dados relativos à assimetria da função auditiva normal, especialização hemisférica e modificações no sistema auditivo central como um resultado da perda de audição, devem ser levados em consideração. Embora o córtex auditivo humano receba eventualmente estímulos sensoriais de ambas as orelhas, é mais fortemente ativado por estimulação da orelha contralateral ${ }^{(18)}$. Portanto, considerando que os centros da fala (Áreas de Broca e Wernicke) estão localizados no córtex cerebral esquerdo em indivíduos destros e podem estar presentes em um ou em ambos os lados em indivíduos canhotos ${ }^{(14)}$, a implantação na orelha direita implicaria em melhores resultados auditivos.

No presente estudo, o lado implantado não interferiu na percepção da fala, apesar de resultados de pacientes implantados na orelha direita apresentarem respostas nos testes de percepção da fala significativamente melhores que os implantados na orelha esquerda ${ }^{(14)}$.

Quanto aos modelos dos aparelhos de implante, não foi verificada qualquer influência sobre os desfechos, o que está de acordo com outros achados ${ }^{(4)}$. Esse é um fator importante, uma vez que envolve custos para aquisição e manutenção do dispositivo, embora não se descartem os critérios estabelecidos pela equipe clínica na escolha dos fabricantes.

Com relação aos resultados pré e pós-implante, todos os pacientes estudados apresentarm melhora no desempenho auditivo após a intervenção, quando comparados às suas capacidades auditivas antes da cirurgia. Os achados assemelham-se aos encontrados na literatura, na qual a média dos limiares auditivos era de 107 dBSPL e apenas 18\% de acertos nos testes de percepção da fala com sentenças, antes do IC. Após o IC, os limiares auditivos passaram a ser de, aproximadamente, $40 \mathrm{dBSPL}$ e os teste de fala com sentenças alcançaram de 60 a $70 \%$ de acerto $^{(2)}$.

Algumas das limitações do presente estudo são inerentes ao próprio desenho do estudo. Por se tratar de um estudo retrospectivo, envolvendo observação por longo período e com informações recuperadas de prontuários médicos, alguns dados clínicos e individuais não estavam disponíveis, comprometendo a análise. Entre essas limitações, estão as informações sobre a audição residual, a habilidade de leitura orofacial, a quantidade de eletrodos inseridos na cóclea, a motivação do paciente e a integridade das vias auditivas centrais. Esses fatores são ainda pouco explorados na literatura, embora existam estudos ${ }^{(6)}$ que evidenciem e sustentem a sua importância como fatores preditores do desempenho auditivo.

\section{CONCLUSÃO}

É possível concluir que o desempenho auditivo após 12 meses de uso do IC supera o critério de indicação na maioria dos indivíduos com perda auditiva pós-lingual, independentemente dos fatores prognósticos avaliados, porém, não em todos os indivíduos pesquisados.

\section{AGRADECIMENTOS}

Agradecemos ao Otocentro-RN e, em especial, à fonoaudióloga Ana Karla Bigois Capistrano pela honrosa colaboração na nossa pesquisa.

\section{REFERÊNCIAS}

1. Moon JI, Kim EY, Jeong JO, Chung W, Cho Y, Hong SH. The influence of various factors on the performance of repetition tests en adults with cochlear implants. Eur Arch Otorhinolaryngol. 2012Mar;269(3):739-45.

2. Manrique M, Ramos A, Morera C, Cenjor C, Lavilla MJ, Boleas MS, et al. Evaluación del implante coclear como técnica de tratamiento de la hipoacusia profunda en pacientes pre y post locutivos. Acta Otorrinolaringol Esp. 2006Jan;57(1):2-23.

3. Bevilacqua MC, Costa AO, Martinho ACF. Implante Coclear. In: Ferreira LP, Befi-Lopes DM, Limongi SCO (Orgs.). Tratado de Fonoaudiologia. São Paulo: Rocca; 2004. p. 751-61.

4. Costa Filho AO, Bevilacqua MC, Amantini, Lamônica Neto D. Implante coclear em adultos. In: Campos CAH, Costa HOO (Orgs.). Tratado de Otorrinolaringologia. São Paulo: Rocca; 2003. p. 278-89.

5. Costa Filho OA, Bevilacqua MC. Implantes Cocleares. In: Costa SS, Cruz OLM, Oliveira JAA. Otorrinolaringologia: princípios e prática. 2a. ed. Porto Alegre: Artes Médicas; 2006. p. 447-53.

6. Oh SH, Kim CS, Kang EJ, Lee DS, Lee HJ, Chang SO, et al. Speech perception after cochlear implantation over 4-year time period. Acta Otolaryngol. 2003Jan;123(2):148-53.

7. Bradley J, Bird P, Monteath P, Wells JE. Improved speech discrimination after cochlear implantation in the Southern Cochlear Implant Adult Programme. N Z Med J. 2010Aug;123(1321):34-44.

8. Padilha Romero MJ, Sainz Quevedo M, Roldán Segura C. Cochlear implant in postlingual adults with progressive hearing loss. Acta Otorrinolaringol Esp. 2004Dec;55(10):457-62.

9. Valente, SLO. Elaboração de listas de sentenças construídas na língua portuguesa [dissertação]. São Paulo (SP): Faculdade de Fonoaudiologia, Pontifícia Universidade Católica; 1998. 
10. Williamson RA, Pytynia K, Oghalai JS, Vrabec JT. Auditory performance after cochlear implantation in late septuagenarians and octogenarians. Otol Neurotol. 2009Oct;30(7):916-20.

11. Klop WM, Boermans PP, Ferrier MB, van den Hout WB, Stiggelbout AM, Frijins JH. Clinical relevance of quality of life outcome in cochlear implantation in postlingually deafened adults. Otol Neurotol. 2008 Aug;29(5):615-21.

12. Hiraumi H, Tsuji J, Kanemaru S, Fujino K, Ito J. Cochlear implants in post-lingually deafened patients. Acta Otolaryngol. 2007Feb;127(s557):17-21.

13. Bevilacqua MC, Moret ALM, Costa OA. Conceituação e indicação do implante coclear. In: Bevilacqua MC, Martinez MAN, Balen AS, Pupo AC, Reis ACMB, Frota S (Orgs.). Tratado de Audiologia. São Paulo: Santos; 2012. p. 407-25.

14. Budenz CL, Cosetti MK, Coelho DH, Birenbaum B, Babb James, Waltzman SB, et al. The effects of cochlear implantation on speech perception in olders adults. J Am Geriatr Soc. 2011Mar;59(3):44653.

15. Hirschfelder A, Gräbel S, Olze H. The impact of cochlear implantation on quality of life: the role of audiologic performance and variables. Otolaryngol Head Neck Surg. 2008Mar;138(3):35762.

16. Cosetti MK, Waltzman SB. Outcomes in cochlear implantation: variables affecting performance in adults and children. Otolaryngol Clin North Am. 2012Feb;45(1):155-71.

17. Migirov L, Taitelbaum-Swead R, Drendel M, Hildesheimer M, Kronenberg J. Cochlear implantation in elderly patients: surgical and audiological outcome. Gerontology. 2010;56(2):123-28.

18. Henkin Y, Taitelbaum-Swead R, Hildesheimer M, Migirov L, Kronenberg J, Kishon-Rabin L. Is there a right cochlear implant advantage? Otol Neurotol. 2008Jun;29(4): 489-94. 